\title{
Fuzzy Integral and Cuckoo Search Based Classifier Fusion for Human Action Recognition
}

\author{
Ilhan AYDIN \\ Department of Computer Engineering, Firat University, Elazig, 23119, Turkey \\ iaydin@firat.edu.tr
}

\begin{abstract}
The human activity recognition is an important issue for sports analysis and health monitoring. The early recognition of human actions is used in areas such as detection of criminal activities, fall detection, and action recognition in rehabilitation centers. Especially, the detection of the falls in elderly people is very important for rapid intervention. Mobile phones can be used for action recognition with their built-in accelerometer sensor. In this study, a new combined method based on fuzzy integral and cuckoo search is proposed for classifying human actions. The signals are acquired from three axes of acceleration sensor of a mobile phone and the features are extracted by applying signal processing methods. Our approach utilizes from linear discriminant analysis (LDA), support vector machines (SVM), and neural networks (NN) techniques and aggregates their outputs by using fuzzy integral. The cuckoo search method adjusts the parameters for assignment of optimal confidence levels of the classifiers. The experimental results show that our model provides better performance than the individual classifiers. In addition, appropriate selection of the confidence levels improves the performance of the combined classifiers.
\end{abstract}

Index Terms-classification, optimization, feature extraction, fuzzy logic, signal processing.

\section{INTRODUCTION}

Activity recognition is a popular research area in the field of human computer interactions. The analysis of human activity is based on the prediction of movement at a specific point in time [1], where a movement consists of actions that occur over time. The recognition of human actions in real time is widely available in many fields (e.g., sport analysis, health monitoring, multimedia, psychology, biology, healthcare). Most researchers have focused on action recognition from video images [2]. Although video images are useful for the film industry and multimedia applications, when considering everyday activity sensors, accelerometers, gyroscopes and Internal Measurement Units (IMU) are preferred [3]. The use of wearable sensors is suitable for this purpose. These wearable sensors are installed directly on the body. However, they may not always be suitable for health services. This is because such sensors are directly attached to the skin and some people can be disturbed by the presence of these sensors [4-5].

A smartphone contains several sensors (e.g., camera, gyroscope, compass, GPS, microphone, light, accelerometer). The data acquired from these sensors can be transmitted to any desired location via wireless devices (e.g., such as Bluetooth, and Wi-Fi) [6-7]. The acceleration sensor is used to detect the user's phone hold on a mobile phone.

This work was supported in part by Firat University Research Project Unit (FUBAP) under Grant MF.16.47.
For example, when you turn a phone, the sensor detects this movement and the user view changes accordingly. This sensor can also be used as a pedometer.

Activity recognition has been used in many areas (e.g., health, advertising, physical therapy, video surveillance). In the health field, treatment progress can be observed by detecting the movements of paralyzed people. Action recognition has also become a necessity for the monitoring of elderly adults living alone and for emergency interventions.

Several methods have been developed for action recognition using an acceleration sensor. The data for the acceleration sensor of the smartphone has been collected for 5 actions [8]. The performance of the artificial neural network, decision tree, and logistic regression for action recognition were compared. The results illustrate that the artificial neural network has a higher performance than the others.

For the early detection of human actions, a hybrid approach has been proposed [9]. The proposed method combines computer vision with fuzzy set theory. The action classification is made by analyzing sequential frames by using fuzzy logic. In some studies, the action was recognized by using data from multiple accelerometers. These studies have aimed to increase the recognition rate of the actions by placing multiple accelerometers at different points on the body [10-11]. Some researchers have combined different sensor data (e.g., gyroscopes, microphones, digital compass) with the acceleration sensor [12-13]. By including the sensor data, it becomes possible to detect more actions with a higher performance ratio.

With the widespread use of mobile smart phones, there is no need for an extra wearable sensor for action recognition. Cho et al. [14] used the sensor of a smart phone to recognize five activities by using a linear discriminant analysis and support vector machine. They acquired signals from three phone sensors: triaxial accelerometer, gyroscope, and magnetometer. Mendez and Casas [15] proposed a nonlinear dynamic system based human action recognition system. The proposed method uses virtual skeleton system points and constitutes a time series of these points. Afterwards, the phase space of these time series is constructed by applying a non-linear time series analysis. Each action is categorized by using support vector machines.

One method for recognizing human actions with the accelerometer and gyroscope sensors found in mobile phones has been proposed [16]. A new feature extraction is performed with the proposed method and the performance of each sensor is provided separately. The experimental 
results revealed that the combination of data from two sensors obtains better than one sensor. Catal et al. [6] used a multiple classifier to recognize daily activities. Their method acquires sensor data for three accelerometers from a smart phone for six actions. The classification of each action was made by combining a logistic regression, artificial neural networks and decision tree results with weighted voting.

In this study, the outputs of three high performance classifiers were combined with the fuzzy integral to recognize human actions. The proposed method uses signals from three accelerometer sensor axes of a smartphone. Signal processing techniques are used to extract the features of the obtained signals. Artificial neural networks, linear discriminant analysis and support vector machines were used for classification purposes. The extracted features are provided to three classifiers, allowing for the output of each classifier to be obtained. The classification results are combined with the fuzzy integral to improve the recognition performance. The fuzzy integral parameter, which indicates the confidence level of the classifiers, was optimized with the cuckoo search algorithm. The proposed method has two primary advantages. The first advantage is to extract the distinguishing features from the obtained signals. The second is to increase the classification result by using the fuzzy integral and cuckoo search.

\section{FUZZY INTEGRAL AND CUCKOO SEARCH BASED CLASSIFIER FUSION}

The proposed method uses the signals from the accelerometer sensor of a smartphone. The received signals are first provided to the signal processing module to obtain the frequency and time domain features. The obtained features are then provided to the artificial neural network, linear discriminant analysis, and support vector machine classifiers. The model of each classifier is constructed in the training stage. Afterwards, the class label for each classifier is calculated by providing the test data of each classifier during the test phase to the model. The obtained classification results are combined with the fuzzy integral and cuckoo search algorithm to improve classification performance. The proposed system is schematically illustrated in Fig. 1.

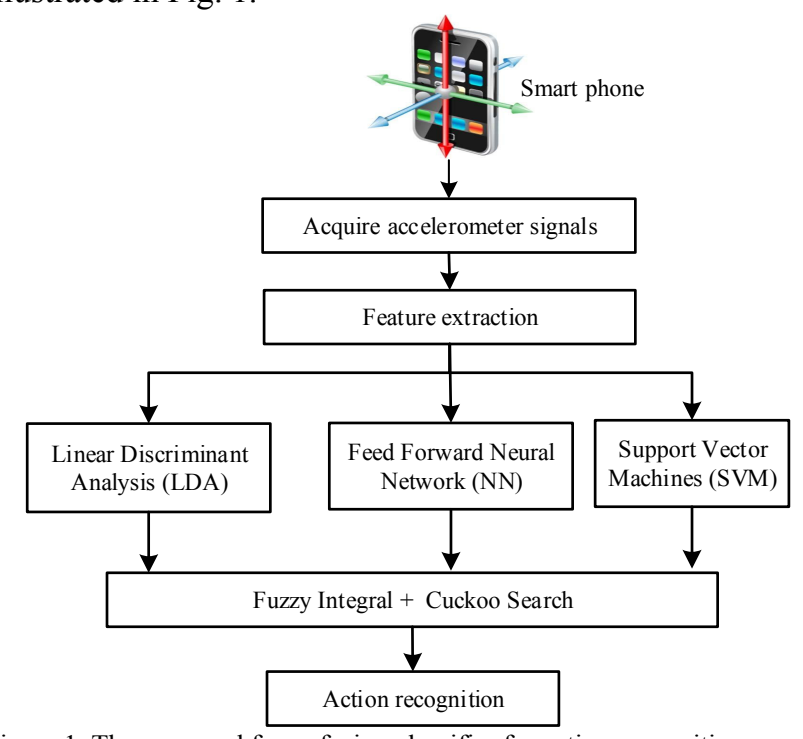

Figure 1. The proposed fuzzy fusion classifier for action recognition
In Fig. 1, three accelerometer signals are acquired from a smartphone. Afterwards, the inputs of the classifiers are obtained by using a feature extraction stage. In this stage, the signal processing based features are extracted. The outputs of the classifiers are provided to the cuckoo search based fuzzy integral and the action is recognized.

\section{A. Signal processing based feature extraction}

For a feature extraction, 66 features are extracted in total: 22 from each axis of the acceleration sensor. The features are obtained for both the time and frequency domain. In the signal pre-processing step, the noise on the signal is removed. The first three feature signals are the RMS values of the three axes. The other three features obtained in the time domain are the mean values of the signals for each axis. For the frequency domain, the spectrum of the signal is first obtained by the Welch method [17]. The steps of the Welch method are presented as follows:

Step 1: First, the original signal X [0..N-1], consisting of $\mathrm{N}$ samples, is divided into $\mathrm{K}$ segments.

Segment 1: $\mathrm{x}[0], \mathrm{x}[1], \ldots, \mathrm{x}[\mathrm{M}-1]$

Segment 2: $x[S], x[S+1], \ldots, x[M-1]$

$\ldots$

Segment K: x[N-M], x[N-M+1], .., x[N-1]

where $\mathrm{M}, \mathrm{S}$, and $\mathrm{K}$ represent the number of samples in each segment, the number of points to shift between segments, and the number of segments, respectively.

Step 2: The windowed discrete Fourier transform for each segment is computed as follows.

$$
X_{k}(v)=\sum_{m} x[m] w[m] \exp (-j 2 \pi v m)
$$

where $\mathrm{m}=(\mathrm{k}-1) \mathrm{S}, \ldots, \mathrm{M}+(\mathrm{k}-1) \mathrm{S}-1$ and $\mathrm{w}[\mathrm{m}]$ is the window function.

Step 3: The modified periodogram value is formed for each segment using a discrete Fourier transform.

$$
P_{k}(v)=\frac{1}{W}\left|X_{k}(v)\right|^{2}, W=\sum_{m=0}^{M} w^{2}[m]
$$

Step 4: To obtain the Welch's estimate from the spectrum, the average of the periodogram value obtained in Step 3 is computed as follows.

$$
S_{k}(v)=\frac{1}{K} \sum_{k=1}^{K} P_{k}(v)
$$

After the spectrum for each axis is obtained by using the Welch method, the position and 6 peak values in the spectrum are determined. Consequently, 12 features are obtained for each accelerometer sensor component. The autocorrelation features are calculated for the three acceleration components. For each component, the height of the main peak, as well as the height and position of the second peak are determined. The last feature involves determining the spectral power features in certain frequency bands adjacent to each other in the spectrum. In this spectrum, the power spectral values of five adjacent frequencies are obtained.

\section{B. Fuzzy integral based classifier fusion}

A fuzzy integral is the fusion of information from different sources during the decision making stage using the objective fuzzy measure. A fuzzy integral is a non-linear function using a fuzzy measure [18]. It has the ability to 
combine information from multiple sources. In the fusion of multiple classifiers, an integral operator combines the information from the classifiers in an objective direction, with respect to certain measures. Combining the results of the classifiers with the fuzzy integral increases the classification performance [19]. In this work, the $g_{\lambda}$-fuzzy integral method proposed by Sugeno is used. The Sugeno integral uses a fuzzy measure in which its range is between 0 and 1. The confidence of each classifier $g$ is chosen according to expert knowledge or problem. In this study, this value is adjusted by the cuckoo search algorithm. The common confidence value $g\left(\mathrm{~A}_{\mathrm{i}}\right)$ of all classifiers is calculated as follows.

$$
\begin{aligned}
& g\left(A_{1}\right)=g\left(\left\{y_{1}\right\}\right)=g^{1} \\
& g\left(A_{i}\right)=g^{i}+g\left(A_{i-1}\right)+\lambda g^{i} g\left(A_{i-1}\right)
\end{aligned}
$$

In (5), $A_{i}$ is a set and is represented by $A_{i}=\left\{y_{1}, y_{2}, \ldots\right.$, $\left.y_{i}\right\}$. Each element of this set indicates the results of the classification for each class. The value $\lambda$ is calculated as follows.

$$
\lambda+1=\prod_{i=1}^{n}\left(1+\lambda g^{i}\right)
$$

In (6), $\lambda \in(-1,+\infty)$ and the non-zero value of $\lambda$ is selected. This parameter can be calculated by solving for a $(\mathrm{n}-1)^{\text {st }}$ degree polynomial.

The calculation of the fuzzy integral only depends on the knowledge of the density function [17]. The density function $\mathrm{g}^{\mathrm{i}}$ indicates the degree of importance $\mathrm{y}$ in relation to the final evaluation. Let $C=\left\{c_{1}, c_{2}, \ldots, c_{k}\right\}$ be a set of classes for the classification problem. Let $Y=\left\{y_{1}, y_{2}, \ldots, y_{n}\right\}$ be a set of classifiers. For our study, we have three classifiers: LDA, $\mathrm{NN}$, and SVM. The parameter $\mathrm{A}$ is an object used as an input for recognition. Let $h_{k}: Y \rightarrow[0,1]$ represent the results of input $A$ for a classifier $y_{k}$. For a class $c_{k}, h_{k}\left(y_{i}\right)$ represents the classification result of classifier $y_{i}$ for class $\mathrm{c}_{\mathrm{k}}$. If the classification result takes one for class $\mathrm{c}_{\mathrm{k}}$, the input A belongs to class $c_{k}$. The $h_{k}\left(y_{i}\right)$ values are sorted in descending order and the density values $\mathrm{g}^{\mathrm{i}}$ are calculated. Afterwards, the class label is determined according to (7).

$$
e=\underbrace{\max }_{i=1 \ldots n}\left[\min \left(h\left(y_{i}\right), g\left(A_{i}\right)\right)\right]
$$

The value $\mathrm{e}$ in (7) illustrates the results of the classification by using a fuzzy integral. There are three classifiers in this study, where the total number of classes in the problem is six. The classifiers calculate the density and the classification result for each class. The obtained values are evaluated according to (7), where the final class of input A will be determined. The classifiers are trained to recognize 6 human actions, and therefore, the number of classes is six. In this study, three classifiers with high performance are used for the recognition of human actions. The overall algorithm of the fuzzy integral based fusion system is provided in Fig. 2.

In Fig. 2, the inputs of the fuzzy integral algorithm are the density level of the classifiers, the training model of each classifier, and an input object of the test data.

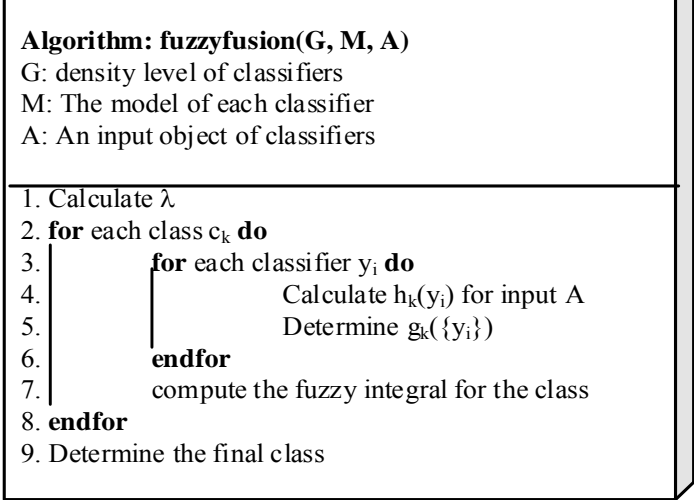

Figure 2. The algorithm of the classifier fusion by the fuzzy integral

LDA is a classification method using a Gaussian distribution [20]. This method calculates the mean vector and covariance matrix of each class to maximize the separability between the distinct classes. The mean of class mean with weighted data is calculated as

$$
\hat{\mu}_{k}=\frac{\sum_{n=1}^{N} M_{n k} w_{n} x_{n}}{\sum_{n=1}^{N} M_{n k}}
$$

where $M_{n k}$ is 1 if the class of $\mathrm{x}_{\mathrm{n}}$ is $\mathrm{k}$. The parameter $\mathrm{w}_{\mathrm{n}}$ represents the positive weight of the data. The covariance matrix is calculated according to (9).

$$
\hat{\Sigma}=\frac{\sum_{n=1}^{N} \sum_{k=1}^{K} M_{n k} w_{n}\left(x_{n}-\hat{\mu}_{k}\right)\left(x_{n}-\hat{\mu}_{k}\right)^{T}}{1-\sum_{k=1}^{K} \frac{W_{k}^{2}}{W_{k}}}
$$

In (9), $W_{k}=\sum_{n=1}^{N} M_{n k} w_{n}$ is the sum of the weights for class $\mathrm{k}$ and $W_{k}^{2}$ is the sum of the squared weights for each class. LDA uses the prior probability, posterior probability, and cost value. The formulation of LDA, in terms of the Bayes rule, is presented in (10).

$$
\hat{y}=\arg \underbrace{\min }_{y=1 . . K} \sum_{k=1}^{K} \hat{P}(k \mid x) C(y \mid k)
$$

In (10), $\hat{y}$ and $\mathrm{K}$ represent the predicted class, and number of classes, respectively. The $\hat{P}(k \mid x)$ and $C(y \mid k)$ are the posterior probability of class $\mathrm{k}$ for observation $\mathrm{x}$ and the cost of an observation $\mathrm{y}$ for class $\mathrm{k}$, respectively. The posterior probability represents the probability of a point $\mathrm{x}$ belonging to class $\mathrm{k}$. It is the product of the prior probability of class $\mathrm{k}(\mathrm{P}(\mathrm{k}))$ and the multivariate normal density. The calculation of this probability is provided in (11).

$$
\begin{aligned}
& \hat{P}(k \mid x)=\frac{1}{\sqrt{2 \pi\left|\Sigma_{k}\right|}} \exp \left(-\frac{1}{2}\left(x-\mu_{k}\right)^{T} \Sigma_{k}^{-1}\right. \\
& \left(x-\mu_{k}\right) P(k)
\end{aligned}
$$

where $\left|\Sigma_{k}\right|$ represents the determinant of the covariance matrix and $\Sigma_{k}^{-1}$ is the inverse of the covariance matrix.

The other classifier, with an output that includes the inputs of the fuzzy integral, is a feed forward artificial 
neural network. For this purpose, a three-layer artificial neural network with a forward feed has been proposed. Overall, 20 neurons are used in the middle layer of the artificial neural network. The artificial neural network is trained by a back propagation learning algorithm [21]. The artificial neural network has 66 inputs and 6 outputs. The structure of the NN is provided in Fig. 3.

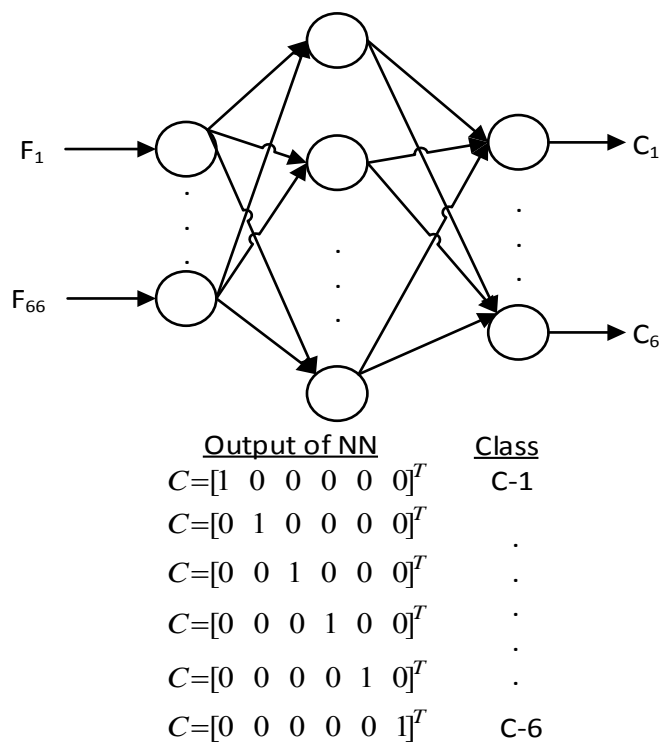

Figure 3. The structure of NN

As shown in Fig. 3, only one of the six outputs of the artificial neural network takes on a value of one; the other outputs take on a value of zero. The output with a value of one represents the actual output. The model is trained by using a backpropagation learning algorithm and updating the weights. The obtained model is used in the test stage to determine the output of a test sample. By using this model, the obtained output for each test sample constitutes one input of the fuzzy integral.

SVM are a two-class classification method. An SVM classifier determines the most appropriate hyperplane to classify the data. The optimal hyperplane is found by maximizing the margin between the two classes [22]. Support vectors are the points closest to the separation plane for each class. The separation hyperplane and the support vectors are illustrated in Fig. 4 for linearly separable data.

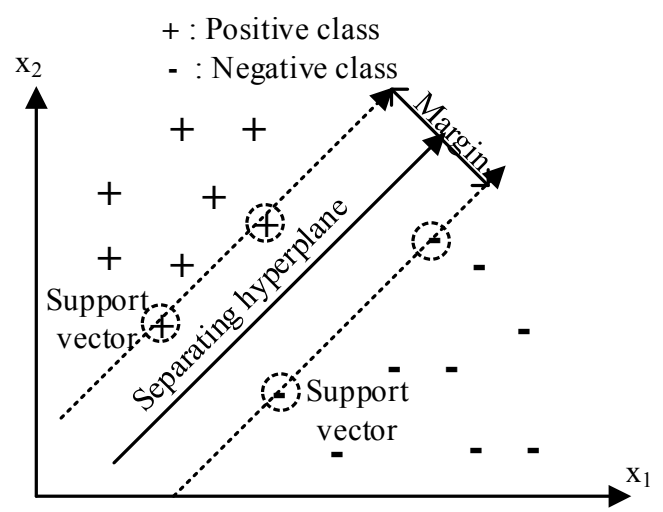

Figure 4. The hyperplane of SVM for linearly separable data

The hyperplane that can be separated linearly is presented in (12).

$$
f(x)=w^{T} x+b=0
$$

In (12), $\mathrm{w}$ is the weight and $\mathrm{b}$ is the constant. The primary purpose of the support vector machine is to minimize $\|\mathrm{w}\|$ and maximize the margin between the two classes. This problem becomes a quadratic optimization problem based on inequality constraints provided by (13).

$$
\begin{aligned}
& \text { Minimize } \frac{1}{2} w^{T} w+c \sum_{i=1}^{n} \xi_{i} \\
& \text { subject to } y_{i}\left(w \phi\left(x_{i}\right)+b\right) \geq 1-\xi_{i}
\end{aligned}
$$

In (13), $y_{\mathrm{i}}$ is the class label where +1 represents a positive class and -1 represents a negative class. The parameter $\xi_{i}$ is a slack variable and indicates a misclassification error. The function $\phi\left(x_{i}\right)$ transforms the input $\mathrm{x}_{\mathrm{i}}$ into a highdimensional space. A control parameter $\mathrm{C}$ should be defined to provide a tradeoff between the minimization of the misclassification rate and the maximization of the margin between the two classes. The problem can be solved by using the Lagrangian multiplier provided in (14).

$$
\begin{aligned}
& \text { Maximize } \sum_{i=1}^{n} \alpha_{i}-\frac{1}{2} \sum_{j=1}^{n} \alpha_{i} \alpha_{j} y_{i} y_{j} K\left(x_{i}, x_{j}\right) \\
& \text { subject to } \sum_{i=1}^{n} \alpha_{i} y_{i}=0,0 \leq \alpha_{i} \leq C
\end{aligned}
$$

where each $\alpha_{i}$ represents a Lagrangian multiplier and its value is greater than zero. The function $\mathrm{K}$ represents the kernel function; the appropriate value of this function increases the accuracy of the classification. The most commonly used kernel functions include linear, tangent hyperbolic, and Gaussian kernel functions. In our study, the Gaussian kernel was used. This kernel function can be presented as

$$
K\left(x_{i}, x_{j}\right)=\exp \left(\left\|x_{i}-x_{j}\right\| / 2 \sigma^{2}\right)
$$

In (15), $\sigma$ represents the radius of the kernel function. After the Lagrangian multiplier and constant $\mathrm{b}$ are found by solving the quadratic optimization problem, the classification is conducted according to the following decision function.

$$
\operatorname{Class}(x)=\operatorname{sgn}\left(\sum_{i=1}^{n} \alpha_{i} y_{i} K\left(x_{i}, x_{j}\right)+b\right)
$$

In (16), the class label is +1 or -1 . In our study, there are a total of 6 classes, so a multiple classification is conducted with the support vector machine. The proposed method uses the one against the other method for a multi-class classification.

\section{Cuckoo search based parameter optimization of fuzzy integral}

The cuckoo algorithm is a new meta heuristic optimization algorithm inspired from the nature [23]. This algorithm is based on the laying strategies of cuckoo birds. A cuckoo bird lays one egg in a randomly chosen nest. The nests with high quality eggs survive and become the next generation of cuckoo birds. The population size is the number of nests. The probability of the cuckoo egg being discovered by the nest owner is $P_{a} \in[0,1]$. If the nest owner becomes aware of a foreign egg, this egg is thrown out of the nest or a new nest is built [24]. This process is the 
replacement of the low-probability eggs with new eggs.

In this study, the cuckoo search algorithm is proposed to adjust the confidence parameters of the fuzzy integral. The proposed method uses the cuckoo search algorithm to obtain the optimum values for the three parameters. Classification performance is obtained by running a fuzzy integral according to each parameter. The classification performance also refers to the fitness of each individual in the population. The proposed algorithm is presented in Fig. 5.

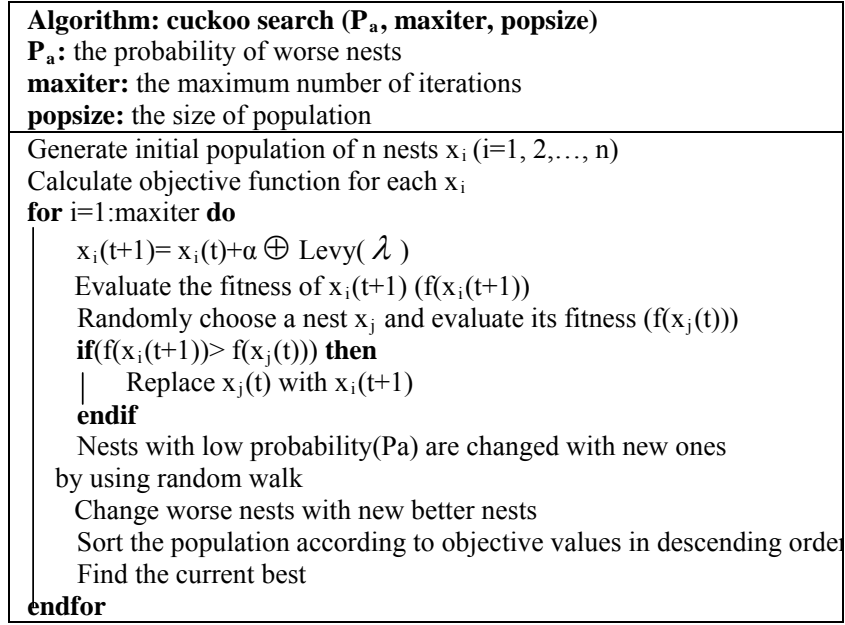

Figure 5. The algorithm of cuckoo search for parameter optimization

In Fig. 5, the parameter $\alpha$ represents the scaling factor of the step size used for a random walk. The product $\oplus$ is an entry wise multiplication. In the search space, the best solution is to search by using the Levy flight for the step size so that it is not bigger than the longest step. In this study, each nest was considered a solution. Each individual has three dimensions and applies the $\mathrm{g}$ parameters associated with each classifier. The search space is fixed between 0 and 1 . The classification rate of the fuzzy integral indicates the objective function.

\section{APPLICATION RESULTS}

In this study, a publicly distributed dataset was used. The activities of 30 individuals were recorded, with each being considered a benchmark dataset [5]. Each condition consists of 10 second segments. The sampling rate of the accelerometer is $50 \mathrm{~Hz}$. Each signal consists of 128 samples. The dataset contains signals for 6 actions: sit, laying, walking upstairs (Upstairs), walking downstairs (Dstairs), walking, and standing. These daily activities can be used as an indicator to determine the functional health of an individual. We used the MATLAB classification learner for the experiments and the high performance classifiers were selected after an extensive number of experiments. The data from the acceleration sensor for each case is presented in Fig. 6. After the original signals are obtained, the feature extraction is performed and the training dataset is constructed. Twenty-two features have been extracted for the signal from each axis of the acceleration sensor. Sixtysix features are obtained in total. In the feature extraction, the spectrum of the signals is obtained by the Welch method and the peak points of the spectrum are detected. The spectra and peak points of the y-axis obtained for three actions are presented in Fig. 7.

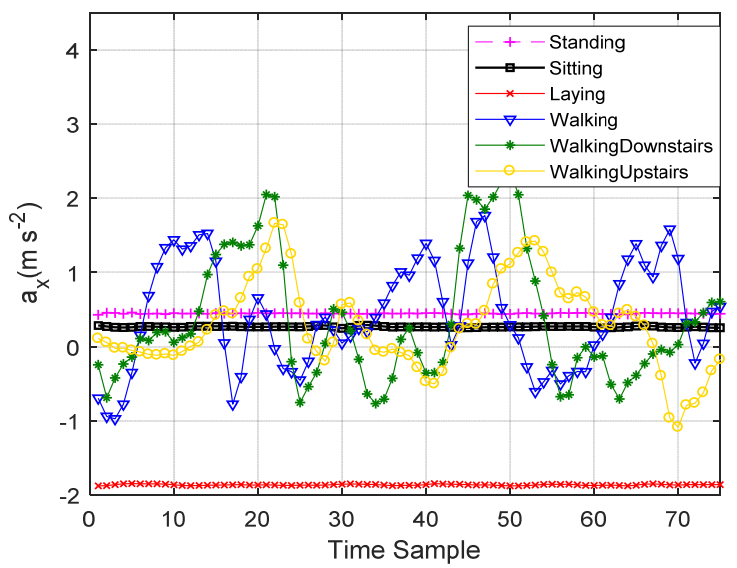

(a) $x$-axis

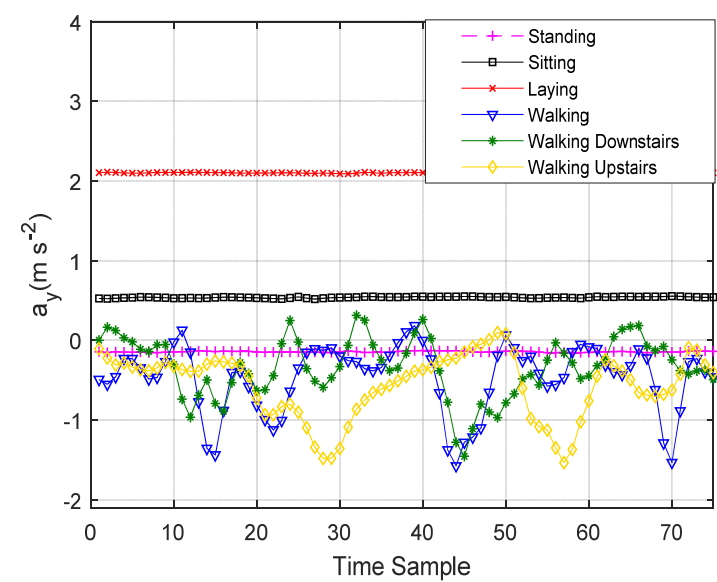

(b) y-axis

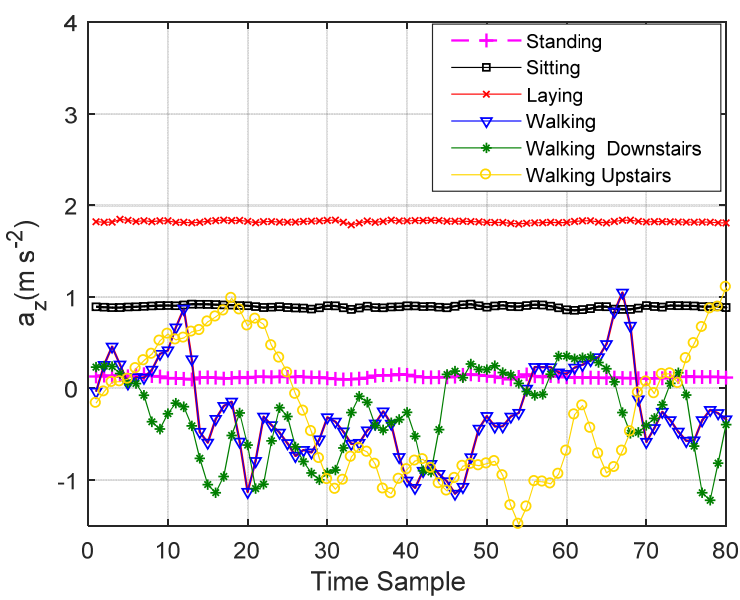

(c) z-axis

Figure 6 . The accelerometer signals of three axis for six actions

As shown in Fig. 7, the spectra of the three actions are different. Although the walking and walking upstairs actions are similar, their peak points can be used to separate two conditions. Instead of considering the entire spectrum, only certain peak points have been chosen as features. In this study, the first six peak points were obtained in the spectrum for each axis. In the fuzzy integral fusion based classification, each classifier is trained using the obtained features and a trained model is constructed. Afterwards, these models are used in the testing stage to obtain an output for a provided input. 


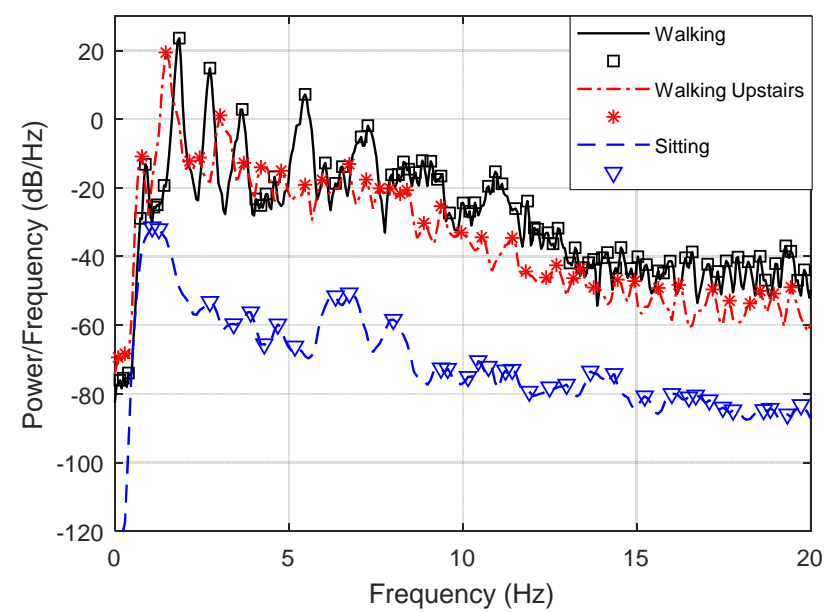

Figure 7. The spectrum and peak points of y-axis for three actions

Our fuzzy integral based activity recognition system was constructed by applying several experiments. The proposed method applies a Sugeno fuzzy integral model for the fusion of the classifiers. The individual classifiers used in the fusion include LDA, NN, and SVM. A tenfold cross validation approach was used in the training stage. Afterwards, the confusion matrix was obtained for each individual classifier. The performance of each classifier and the fuzzy fusion based classifier fusion were evaluated in terms of the precision, recall, F1 score, and accuracy rate.

To evaluate the effectiveness of the proposed method, we conduct some calculations on the confusion matrix. The confusion matrix, with actual and predicted outputs, is used to evaluate the performance of the classifiers. In the confusion matrix, four measures are used to illustrate the performance of the classifier: precision, recall, accuracy, and the F1 measure. Table I illustrates an example of a confusion matrix for a multiple classifier.

TABle I. AN EXAMPLE OF CONFUSION MATRIX FOR MUltiPle Classifier

\begin{tabular}{|c|c|c|c|c|c|}
\hline & \multicolumn{4}{|c|}{ Actual Class } \\
\hline & & 1 & 2 & 3 & Sum \\
\hline \multirow{4}{*}{ 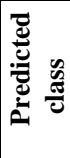 } & 1 & $\mathbf{T P}_{11}$ & $\mathrm{FP}_{12}$ & $\mathrm{FP}_{13}$ & $\mathrm{NI}_{1}$ \\
\hline & 2 & $\mathrm{FP}_{21}$ & $\mathbf{T P}_{22}$ & $\mathrm{FP}_{23}$ & $\mathrm{NI}_{2}$ \\
\hline & 3 & $\mathrm{FP}_{31}$ & $\mathrm{FP}_{32}$ & $\mathbf{T P}_{33}$ & $\mathrm{NI}_{3}$ \\
\hline & Sum & $\mathrm{NT}_{1}$ & $\mathrm{NT}_{2}$ & $\mathrm{NT}_{3}$ & total \\
\hline
\end{tabular}

The precision represents the measure of the relevancy of results. Precision is the ratio of the number of positively predicted samples to the total number of positively classified samples. If a classification problem has $\mathrm{C}$ classes, the precision can be calculated by using (17).

$$
\text { Precision }=\frac{1}{C} \sum_{i=1}^{C} \frac{T P_{i i}}{N I_{i}}
$$

where $\mathrm{TP}_{\mathrm{ii}}$ and $\mathrm{NI}_{\mathrm{i}}$ represent the number of correctly classified samples for class $i$ and the total number of test samples that are classified as label $\mathrm{i}$, respectively. The parameter $\mathrm{NI}_{i}$ is the sum of the number in row $i$. The second parameter is recall, which is the ratio of correctly classified samples for class $i$ to the total number of samples for class $i$ labels. This parameter is calculated by using (18).

$$
\text { Recall }=\frac{1}{C} \sum_{i=1}^{C} \frac{T P_{i i}}{N T_{i}}
$$

In (18), $\mathrm{NT}_{\mathrm{i}}$ represents the test samples with true label I, which can be calculated by summing the corresponding column. The third parameter is the F1 score, which can be defined as the harmonic mean of precision and recall. F1 takes on a value between 0 and 1, where 1 means that all test samples are correctly classified. This parameter is calculated by using (19).

$$
F 1=2 * \frac{\text { precision } * \text { recall }}{\text { precision }+ \text { recall }}
$$

The last parameter is accuracy, which refers to the number of samples that are correctly classified. The accuracy rate is calculated as follows.

$$
\text { Accuracy }=\frac{\sum_{i=1}^{C} T P_{i i}}{\text { total }}
$$

LDA is a classifier which uses a multivariate normal distribution based on the Gaussian model. While the means for each class varies, the covariance remains the same. The confusion matrix is presented in Table II for LDA.

TABLE II. THE ACCURACY RATE OF LDA FOR ACTION RECOGNITION

\begin{tabular}{|c|c|c|c|c|c|c|}
\hline & Walking & Upstairs & Dstairs & Sit & Stand & Laying \\
\hline Walking & 1629 & 74 & 19 & 0 & 0 & 0 \\
\hline Upstairs & 82 & 1386 & 76 & 0 & 0 & 0 \\
\hline Dstairs & 65 & 122 & 1219 & 0 & 0 & 0 \\
\hline Sit & 1 & 0 & 0 & 1192 & 569 & 15 \\
\hline Stand & 0 & 1 & 0 & 147 & 1758 & 0 \\
\hline Laying & 0 & 0 & 0 & 0 & 0 & 1944 \\
\hline
\end{tabular}

In Table II, the sitting and downstairs actions decrease the overall performance of the classifier. SVM is a two-class classification method. Since a multi-class classification was made in this study, one versus the other method was used for the multi-class classification. The used classification method is based on error correcting output codes. This method reduces the multiple classification problem to a set of binary classifiers. The Gaussian function is used as the kernel function. The confusion matrix is presented in Table III for SVM.

TABLE III. THE ACCURACY RATE OF SVM FOR ACTION RECOGNITION

\begin{tabular}{|c|c|c|c|c|c|c|}
\hline & Walking & Upstairs & Dstairs & Sit & Stand & Laying \\
\hline Walking & 1638 & 45 & 39 & 0 & 0 & 0 \\
\hline Upstairs & 57 & 1437 & 50 & 0 & 0 & 0 \\
\hline Dstairs & 41 & 78 & 1287 & 0 & 0 & 0 \\
\hline Sit & 0 & 1 & 0 & 1484 & 287 & 5 \\
\hline Stand & 0 & 0 & 1 & 219 & 1686 & 0 \\
\hline Laying & 0 & 0 & 0 & 1 & 0 & 1943 \\
\hline
\end{tabular}

According to Table III, the SVM-based classification method provides better results than the LDA. However, for some classes, the performance of the SVM is lower than that of the LDA. In particular, there was a significant decrease in the misclassified data in the sitting class. However, in other classes, the number of misclassified samples increased. In Table IV, the action recognition results are provided by applying NN. 
TABLE IV. THE ACCURACY RATE OF NN FOR ACTION RECOGNITION

\begin{tabular}{|c|c|c|c|c|c|c|}
\hline & Walking & Upstairs & Dstairs & Sit & Stand & Laying \\
\hline Walking & 1550 & 64 & 106 & 1 & 1 & 0 \\
\hline Upstairs & 65 & 1375 & 104 & 0 & 0 & 0 \\
\hline Dstairs & 69 & 88 & 1249 & 0 & 0 & 0 \\
\hline Sit & 0 & 3 & 0 & 1570 & 204 & 0 \\
\hline Stand & 0 & 1 & 0 & 153 & 1752 & 0 \\
\hline Laying & 0 & 0 & 0 & 0 & 0 & 1944 \\
\hline
\end{tabular}

A three layered feedforward artificial neural network was used to obtain the results in Table III. The artificial neural network has 66 inputs and 6 outputs. In the middle layer of the network, 18 nodes were used. The overall performance is lower than SVM, although the classification performance with artificial neural networks generally increased. In Table $\mathrm{V}$, the results of combining the performance of the three methods with the fuzzy integral are provided.

TABLE V. THE ACCURACY RATE OF FUZZY INTEGRAL FOR ACTION RECOGNITION

\begin{tabular}{|c|c|c|c|c|c|c|}
\hline & Walking & Upstairs & Dstairs & Sit & Stand & Laying \\
\hline Walking & 1550 & 64 & 106 & 1 & 1 & 0 \\
\hline Upstairs & 65 & 1375 & 104 & 0 & 0 & 0 \\
\hline Dstairs & 69 & 88 & 1249 & 0 & 0 & 0 \\
\hline Sit & 0 & 3 & 0 & 1570 & 204 & 0 \\
\hline Stand & 0 & 1 & 0 & 153 & 1752 & 0 \\
\hline Laying & 0 & 0 & 0 & 0 & 0 & 1944 \\
\hline
\end{tabular}

In Table $\mathrm{V}$, the fuzzy integral fusion outperformed each individual classifier. Not only did it yield higher AUC values, it also resulted in a higher accuracy rate. The confidence level of each classification is equal. This illustrates the same effect of each individual classifier on the classification. In this study, the weights of the classifiers are adjusted by applying the cuckoo search algorithm, while the parameters of the cuckoo algorithm are presented in Table VI.

TABLE VI. THE PARAMETERS OF CUCKOO SEARCH ALGORITHM
\begin{tabular}{|c|c|}
\hline Parameter & Value \\
\hline Population size & 25 \\
\hline $\mathrm{Pa}$ & 0.25 \\
\hline Maxiter & 200 \\
\hline$\alpha$ & 1 \\
\hline$\lambda$ & 2.5 \\
\hline
\end{tabular}

The parameters presented in Table VI were obtained by running the Cuckoo search algorithm 10 times. The best performance was achieved with these parameters. The fitness function of the Cuckoo search algorithm is the maximization of the classification performance. The average convergence performance of the Cuckoo search algorithm is presented in Fig. 8.

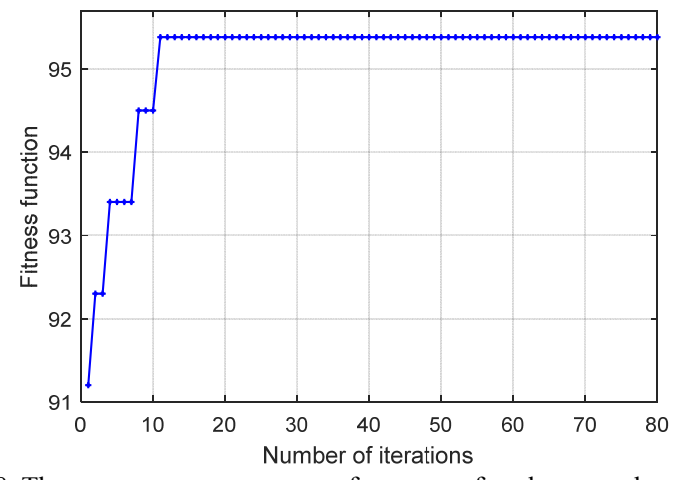

Figure 8 . The average convergence performance of cuckoo search
As shown in Fig. 8, the performance of the Cuckoo search algorithm reaches a maximum performance, on average, of 12 steps. The classification performance was taken as the fitness function and a maximum performance of $95.38 \%$ was achieved. The results of the fuzzy integral and cuckoo search based fusion are presented in Table VII.

TABLE VII. THE ACCURACY RATE OF FUZZY INTEGRAL AND CUCKOO SEARCH BASED FUSION FOR ACTION RECOGNITION

\begin{tabular}{|c|c|c|c|c|c|c|}
\hline & Walking & Upstairs & Dstairs & Sit & Stand & Laying \\
\hline Walking & 1699 & 3 & 19 & 0 & 0 & 1 \\
\hline Upstairs & 13 & 1507 & 24 & 0 & 0 & 0 \\
\hline Dstairs & 15 & 38 & 1353 & 0 & 0 & 0 \\
\hline Sit & 0 & 1 & 1 & 1492 & 281 & 2 \\
\hline Stand & 0 & 0 & 1 & 268 & 1637 & 0 \\
\hline Laying & 0 & 1 & 1 & 8 & 0 & 1934 \\
\hline
\end{tabular}

As illustrated in Table VII, after the cuckoo search is applied to the weights of each classifier, the accuracy rate of the fusion method will exhibit improvements. The results of the confusion matrix were used to determine and evaluate the performance of each classifier according to four criteria. The comparison results are presented in Table VIII for the LDA, SVM, fuzzy integral (FI), and fuzzy integral and cuckoo search (FICS).

TABLE VIII. PERFORMANCE COMPARISON OF CLASSIFIERS
\begin{tabular}{|c|c|c|c|c|}
\hline Classifier & Precision & Recall & F1 & Accuracy \\
\hline LDA & 89.33 & 88.40 & 88.85 & 88.63 \\
\hline SVM & 92.03 & 91.94 & 91.98 & 92.00 \\
\hline NN & 91.34 & 91.36 & 91.35 & 91.66 \\
\hline FI & 93.65 & 93.64 & 93.64 & 93.43 \\
\hline FICS & 95.38 & 95.37 & 95.38 & 95.30 \\
\hline
\end{tabular}

In Table VIII, we observe that the fusion of the classifier consistently obtains better performance than that of the individual classifiers for all performance measures. The best performance among the individual classifiers is achieved by using support vector machines. When the classification results are combined by using the fuzzy integral, over $93 \%$ of the performance is achieved for all of the metrics. When the parameters of the fuzzy integral are optimized with the cuckoo search, the performance is obtained as $95.3 \%$ for all of the metrics. The proposed method is compared with other machine learning results in the literature on the same dataset [5-6]. The results are presented in Fig. 9.

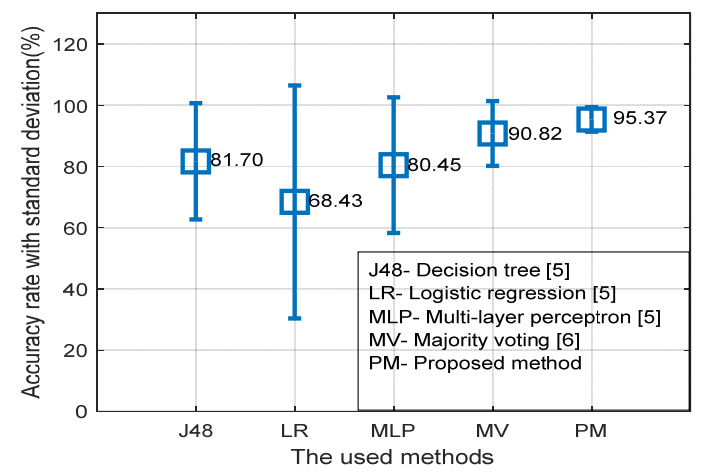

Figure 9. The comparison results of the proposed method with other conventional methods

According to Fig. 9, our proposed model provides better results than other conventional classification methods. With the developed method, the recognition rates of the downstairs and upstairs actions increased. Consequently, 
two basic contributions to the literature in the field of human action recognition have been made in this study. The first contribution is the distinguishing properties extracted by the spectral-based preprocessing methods. The second contribution is to select the weights of each classifier according to their performance on classification. With the developed method, the classification performance is improved, while the standard deviation is reduced.

\section{CONCLUSION}

Activity recognition is an important research area, where a small change in performance causes a significant influence. Activity recognition is especially used in the health and rehabilitation areas. The accurate detection of the action is of great importance in order to provide intervention and treatment possibilities in the case of illness. Previous studies have primarily focused on attempting to define actions with a combination of sensors. In recent years, the use of sensors in mobile phones, for different purposes in the health field, has become widespread.

In this study, the signals from the acceleration sensor of the smartphone were used for motion recognition. We propose a novel ensemble method with a cuckoo search optimized fuzzy integral fusion for action recognition. The proposed method combines the results of three classification methods with a high classification performance: LDA, SVM, and NN. These classifiers were used in the fuzzy fusion algorithm and the appropriate class was determined according to the incoming sample to the fuzzy integral. All experiments were performed on a publically available dataset. The experimental results illustrate that the fusion of good classifiers improves the action recognition performance.

\section{REFERENCES}

[1] M. Vrigkas, V. Karavasilis, C. Nikou, \& I.A. Kakadiaris, "Matching mixtures of curves for human action recognition," Computer Vision and Image Understanding, vol. 119, pp. 27-40, Feb. 2014, doi:10.1016/j.cviu.2013.11.007

[2] J. Morales, D. Akopian, "Physical activity recognition by smartphones, a survey,". Biocybernetics and Biomedical Engineering, vol. 37, pp. 388-400, May 2017. doi:10.1016/j.bbe.2017.04.004

[3] L. Bao, S. Intille, "Activity recognition from user-annotated acceleration data," Pervasive computing, vol. 3001, pp. 1-17, Apr 2004, doi:10.1007/978-3-540-24646-6 1

[4] L. Chen, J. Hoey, C.D. Nugent, D.J. Cook, Z. Yu, "Sensor-based activity recognition," IEEE Transactions on Systems, Man, and Cybernetics, Part C, vol. 42, pp. 790-808, May 2012, doi:10.1109/TSMCC.2012.2198883

[5] J.R. Kwapisz, G. M. Weiss, S.A. Moore, "Activity recognition using cell phone accelerometers," ACM SigKDD Explorations Newsletter, vol. $\quad 12, \quad$ pp. 74-82, Dec. 2011 , doi:10.1145/1964897.1964918

[6] G. Son, S. Kwon, Y. Lim, "Speech Rate Control for Improving Elderly Speech Recognition of Smart Devices," Advances in Electrical and Computer Engineering, vol.17, no.2, pp.79-84, May 2017, doi:10.4316/AECE.2017.02011
[7] C. Catal, S. Tufekci, E. Pirmit, G. Kocabag, G. "On the use of ensemble of classifiers for accelerometer-based activity recognition," Applied Soft Computing, vol. 37, pp. 1018-1022, Dec. 2015, doi:10.1016/j.asoc.2015.01.025

[8] M. Field, D. Stirling, Z. Pan, M. Ros, F. Naghdy, "Recognizing human motions through mixture modeling of inertial data," Pattern Recognition, vol. 48, pp. 2394-2406, Aug. 2015 , doi:10.1016/j.patcog.2015.03.004

[9] E. Vats, C. S. Chan, "Early detection of human actions-a hybrid approach," Applied Soft Computing, vol. 46, pp. 953-966, Sept. 2016 , doi:. 10.1016/j.asoc.2015.11.007

[10] F. Attal, S. Mohammed, M. Dedabrishvili, F. Chamroukhi, L. Oukhellou, Y. Amirat, "Physical human activity recognition using wearable sensors," Sensors, vol. 15, 31314-31338, Dec. 2015, doi:10.3390/s151229858

[11] A. Mannini, A.M. Sabatini, "Machine learning methods for classifying human physical activity from on-body accelerometers," Sensors, vol. 10, pp. 1154-1175, Feb. 2010. doi: $10.3390 / \mathrm{s} 100201154$

[12] O.-A. Schipor, S.-G. Pentiuc, M.-D. Schipor, "Toward automatic recognition of children's affective state using physiological parameters and fuzzy model of emotions," Advances in Electrical and Computer Engineering, vol.12, pp.47-50, May 2012, doi:10.4316/AECE.2012.02008

[13] L. Gao, A.K. Bourke, J. Nelson, "Activity recognition using dynamic multiple sensor fusion in body sensor networks," In: Proc of IEEE Engineering in Medicine and Biology Society, San Diego, 2012, pp. 1077-1080, doi:10.1109/EMBC.2012.6346121

[14] K. Cho, N. Iketani, H. Setoguchi, M. Hattori, M. "Human activity recognizer for mobile devices with multiple sensors," In: IEEE International Conference on Ubiquitous, Autonomic and Trusted Computing, 2009, pp. 114-119, doi:10.1109/UIC-ATC.2009.51

[15] A. López-Méndez, J.R. Casas, "Model-based recognition of human actions by trajectory matching in phase spaces," Image and Vision Computing, vol. 30, pp. 808-816, Nov. 2012, doi:10.1016/j.imavis.2012.06.007

[16] A. Wang, G. Chen, J. Yang, S. Zhao, C.Y. Chang, "A comparative study on human activity recognition using inertial sensors in a smartphone," IEEE Sensors Journal, vol. 16, pp.4566-4578, March 2016, doi:10.1109/JSEN.2016.2545708

[17] K. Barbe, R. Pintelon, J. Schoukens. "Welch method revisited: nonparametric power spectrum estimation via circular overlap," IEEE Transactions on signal processing, vol. 58, pp. 553-565, Feb. 2010, doi:10.1109/TSP.2009.2031724

[18] S.B. Cho, J.H. Kim, "Multiple network fusion using fuzzy logic," IEEE Transactions on Neural Networks, vol. 6, pp. 497-501, Mar 1995, doi:10.1109/72.363487

[19] S. L. Wu, Y.T. Liu, T.Y. Hsieh, Y.Y. Lin, C.Y. Chen, C.H. Chuang, C. T. Lin, "Fuzzy integral with particle swarm optimization for a motor-imagery-based brain-computer interface," IEEE Transactions on Fuzzy Systems, vol. 25, pp. 21-28, Aug 2016, doi:10.1109/TFUZZ.2016.2598362

[20] J. Friedman, T. Hastie, R. Tibshirani, The elements of statistical learning, New York: Springer series in statistics, pp. 241-249, 2001.

[21] V.V. Phansalkar, P. S. Sastry "Analysis of the back-propagation algorithm with momentum," IEEE Transactions on Neural Networks vol. 5, pp. 505-506, May 1994, doi:10.1109/72.286925

[22] N. Cristianini, B. Scholkopf, "Support vector machines and kernel methods: the new generation of learning machines," $\mathrm{A}$ Magazine, vol. 23, pp. 31-41, Fall 2002, doi:10.1609/aimag.v23i3.1655

[23] R. Rajabioun, "Cuckoo optimization algorithm," Applied soft computing, vol. 11, pp. 5508-5518, Dec. 2011, doi:10.1016/j.asoc.2011.05.008.

[24] A.H. Gandomi, X.S. Yang, A.H. Alavi, "Cuckoo search algorithm: a metaheuristic approach to solve structural optimization problems," Engineering with computers, vol.29, pp. 17-35, Jan. 2013 , doi:10.1007/s00366-011-0241-y 